\title{
LA ESFERA PÚBLICA ÁRABE Y LAS RELACIONES EXTERIORES DE CATAR DESDE 1995
}

\author{
THE ARAB PUBLIC SPHERE \\ AND QATARI FOREIGN RELATIONS \\ SINCE 1995
}

\author{
LA SPHÈRE PUBLIQUE ARABE \\ ET LES AFFAIRES ÉTRANGÈRES DU QATAR \\ DEPUIS 1995
}

\author{
Erick Viramontes Viramontes \\ Universidad Nacional Australiana \\ erick.viramontes@anu.edu.au
}

Resumen: Según las perspectivas más recientes sobre la política exterior de los Estados árabes, en este artículo se sostiene que ciertos rasgos intrigantes de las relaciones exteriores de Catar desde mediados de los años noventa son prueba del influjo de la esfera pública árabe en la toma de decisiones de este Estado, ya que demuestran los intentos del liderazgo catarí por posicionarse en esa esfera pública por medio de iniciativas con gran valor simbólico. A partir del análisis de reportajes periodísticos y de entrevistas a los principales líderes de Catar por medios reconocidos internacionalmente, este trabajo hace una aportación a los debates sobre diplomacia en el Medio Oriente y multiplica la bibliografía hispánica sobre un Estado cuya relevancia ha aumentado considerablemente en las primeras décadas de la centuria que corre ahora.

Palabras clave: Catar; Relaciones Exteriores; esfera pública árabe; $\mathrm{Al}$ Thani; Medio Oriente.

ABSTRACT: According to the most recent perspectives on the foreign policy of the Arab States, this article argues that certain intriguing features of Qatar's foreign relations since the mid-1990s are proof of the influence of the Arab public sphere in decision-making by this State, as they demonstrate the attempts of the Qatari leadership to position itself in that public sphere through initiatives that hold great symbolic value. Based on an analysis of media re- 
ports and interviews with the principal leaders of Qatar by internationally recognized media outlets, this research makes a contribution to the debates on diplomacy in the Middle East and expands the Spanish-language bibliography on a State whose importance has increased considerably in the first two decades of the current century.

Keywords: Qatar; Foreign Relations; Arab public sphere; Al Thani; Middle East.

\section{Traducción de Gonzalo Celorio Morayta}

RÉSumÉ: Sous l'éclairage des analyses les plus récentes concernant la politique étrangère des États arabes, l'article propose que certains traits particuliers de celle du Qatar, depuis le milieu des années 1990, prouvent l'influence de la sphère publique arabe sur les décisions de ce pays, car ils mettent en évidence les efforts des dirigeants qataris pour s'affirmer dans cette sphère au moyen d'initiatives qui revêtent une grande valeur symbolique. En s'appuyant sur l'analyse de reportages journalistiques, ainsi que d'entretiens des principaux dirigeants du Qatar avec les médias internationaux les plus renommés, l'article tente de contribuer au débat sur la diplomatie au Moyen Orient. Il s'ajoute ainsi à la bibliographie en espagnol sur un pays dont l'importance a augmenté considérablement le long des deux premières décennies du siècle.

Mots clefs: Qatar; Relations Internationales; sphère publique arabe; $\mathrm{Al}$ Thani; Moyen Orient.

\section{Traducción de BERNARDo MABIRE}

Fecha de recepción: octubre de 2017

Fecha de aceptación: julio de 2018 
$\mathrm{C}$ ON base en discusiones recientes sobre la política exterior de los Estados árabes, en este artículo se propone un marco conceptual para entender las relaciones exteriores de Catar durante los gobiernos de Hamad bin Jalifa Al Thani, quien llegó al poder por medio de un golpe de Estado en 1995, y de su hijo, Tamim bin Hamad Al Thani, quien lo sustituyó como emir de Catar, en junio de 2013. A más de tomar en cuenta los esfuerzos por garantizar la seguridad de un Estado pequeño situado en un contexto turbulento, aquí se propone prestar atención a la esfera pública árabe y al interés del liderazgo catarí por mejorar su posición y reputación regionales mediante iniciativas con un alto valor simbólico en esa esfera pública.

El argumento que guía este artículo privilegia los debates en la esfera pública árabe como factores que informan e influyen las relaciones exteriores de los Estados del Medio Oriente. En otras palabras, mediante el análisis de la proyección internacional de Catar, es posible observar la forma en que la toma de decisiones en el ámbito de las relaciones exteriores entre las estructuras estatales interactúa con las tendencias en una esfera pública trasnacional compuesta de plataformas tecnológicas de comunicación. En Catar, el influjo de la esfera pública árabe en el proceso de toma de decisiones se manifiesta en el lanzamiento de iniciativas con alto valor simbólico, como rechazar presiones para cerrar o cambiar la línea editorial de la televisora Al-Jazeera, cuya cobertura ha provocado numerosas controversias; otorgar apoyo constante a organizaciones consideradas como terroristas por parte de sus aliados internacionales, no menos que asilo a los opositores políticos de varios Estados vecinos, lo que ha provocado tensiones entre el gobierno catarí y los regímenes de la región. Según se evidencia líneas abajo, la manifestación concreta de la esfera pública árabe en las relaciones exteriores de Catar se debe a ciertas características propias.

Este trabajo pretende hacer una doble aportación. Por un lado, busca involucrarse en los debates sobre política exterior 
de los Estados árabes, prestando atención a un caso ignorado por tales aproximaciones, como el de las relaciones exteriores de Catar. ${ }^{1}$ Por otro, brindar elementos para entender las relaciones exteriores de un Estado, cuyo creciente activismo en los ámbitos regional e internacional ha atraído la atención del mundo. A pesar de que la bibliografía sobre Catar se ha multiplicado en sincronía con la relevancia de ese Estado del Golfo, según unos, Árabe o, según otros, Pérsico, ${ }^{2}$ las fuentes en español no menudean. ${ }^{3}$

Para exponer el argumento descrito arriba, el presente artículo se divide en cuatro secciones. En la primera, se discute por qué se tienen ciertas iniciativas diplomáticas recientes por rasgos intrigantes de las relaciones exteriores de Catar. En la segunda, se analizan los distintos argumentos que campean en la bibliografía con respecto de esas iniciativas. En una explicación más completa del principio según el cual este artículo se articula consiste la tarea de la tercera parte. Se analizan, en la cuarta, los rasgos intrigantes de las relaciones exteriores cataríes a la luz del argumento discutido en la sección anterior. Este trabajo se basa en fuentes secundarias como reportajes periodísticos sobre desarrollos políticos con-

${ }^{1}$ Sobre la política exterior de los Estados árabes y del Medio Oriente, véase R. Hinnebush y A. Енteshami (eds.), The Foreign Policies of Middle East States, Londres, Lynne-Rienner, 2002; S. Telhami y M. BarnetT (eds.), Identity and Foreign Policy in the Middle East, Ithaca, Cornell University Press, 2002; B. Korany y A. E. H. Dessouki (eds.), The Foreign Policies of Arab States: The Challenge of Globalization, Cairo, The American University in Cairo Press, 2008.

${ }^{2}$ Por la controversia en torno a la denominación de la porción de agua que se encuentra entre Irán y la península arábiga, se la va a denominar en estas páginas tan sólo como el Golfo.

${ }^{3}$ Algunos trabajos recientes en castellano sobre Catar son los siguientes: S. KaWAKIBI, "Al-Yazira y la política exterior de Qatar", Awraq. Revista de Análisis y Pensamiento sobre el Mundo Árabe e Islámico Contemporáneo, 2010, núm. 2, pp. 61-78; E. Woertz, "Qatar y el descuido europeo de la región del Golfo Pérsico", en Notes Internationals CIDOB, 2012, núm. 46, pp. 1-6; A. Priego, "Las Primaveras Árabes: la influencia de Qatar y sus relaciones con los estados del Golfo", Revista UNISCI, 2015, núm. 39, pp. 233-252. 
temporáneos en el Medio Oriente que involucran a Catar, así como entrevistas con los principales líderes del país, publicadas en medios noticiosos reconocidos en el ámbito internacional. Estos documentos fueron recopilados por medio de una búsqueda exhaustiva en la base de datos FACTIVA, durante el período en que esta investigación se concentra.

\section{RASGOS INTRIGANTES}

DE LAS RELACIONES EXTERIORES DE CATAR

Desde su independencia en 1971, cuando el llamado Imperio informal británico en el este de la península arábiga ${ }^{4}$ llegó a su fin, el Estado de Catar ha enfrentado retos similares a los de los Estados vecinos, como los de la dificultad de mantener la integridad territorial con una capacidad defensiva incipiente en un entorno marcado por la ambigüedad en la demarcación fronteriza, las ambiciones expansionistas de los distintos gobiernos en la región y la existencia de identidades trasnacionales que dificultan la constitución de una identidad nacional. ${ }^{5}$ Catar, por tanto, ha seguido un patrón predecible en su actividad diplomática, la cual ha privilegiado el acercamiento a los poderes occidentales, especialmente Estados Unidos, desde principios de los años noventa, y el apoyo a las iniciativas regionales de Arabia Saudita y del resto de los Estados del Consejo de Cooperación del Golfo (CCG). ${ }^{6}$ Las relaciones

${ }^{4}$ El rótulo "Imperio informal británico en el este de la península arábiga" fue ideado por el historiador James Onley para describir el sistema de relaciones entre la corona británica y los liderazgos locales en la región del Golfo. A este respecto, véase J. ONLEY, The Arabian Frontier of the British Raj: Merchants, Rulers, and the British in the Nineteen-Century Gulf, Nueva York, Oxford University Press, 2007.

${ }^{5}$ Para una discusión más detallada acerca de los retos para los Estados del Golfo desde su independencia, véase G. Gause, The International Relations of the Persian Gulf, Cambridge, University Press, 2010, pp. 9-11.

${ }^{6} \mathrm{El} 25$ de mayo de 1981, en medio de la prolongada guerra entre Irak y la recién proclamada República Islámica de Irán, surgió el Consejo de Cooperación del Golfo como una organización regional que aglutinaba a 
exteriores de Catar parecen grosso modo bastante predecibles y coincidentes con las posibilidades abiertas a los nuevos Estados árabes del Golfo después de su independencia.

Sin embargo, la diversificación en las relaciones exteriores de Catar desde mediados de los años noventa ha incluido un entramado complejo de iniciativas intrigantes que se antoja no pocas veces contradictorio. Desde la ascensión al poder de Hamad bin Jalifa Al Thani, luego de haber dirigido un golpe de Estado palaciego en contra de su padre en junio de 1995, Catar ha sufrido importantes transformaciones en todos los ámbitos. Entre otras cosas, el nuevo gobierno comenzó la explotación de sus grandes reservas de gas en el yacimiento del norte, convirtiéndose así en una potencia exportadora de gas natural licuado. ${ }^{7}$ Asimismo, la sucesión del poder dio paso a un proceso moderado de reforma política interna, considerado por algunos como un conjunto de cambios cosméticos. ${ }^{8}$ Estos cambios se acompañaron de un incremento notorio en la actividad diplomática del gobierno catarí en los ámbitos regional e internacional, involucrándose en esfuerzos de mediación de distintos conflictos entre países vecinos y haciendo las veces de sede para actos de envergadura mundial, como la ya no tan lejana edición de la Copa Mundial de Futbol del año 2022.

En el ámbito diplomático, el gobierno de Catar ha lanzado una serie de iniciativas que le granjeó un carácter complejo y multidimensional. La primera de éstas ha sido la apertura

los Estados de Arabia Saudita, Bahréin, Kuwait, Omán, Catar y Emiratos Árabes Unidos. Desde sus inicios, el cCG incluyó un esquema militar de cooperación conocido como Peninsula Shield Force y, más recientemente, se ha expandido para incluir proyectos de integración económica, como la paulatina creación de una unión aduanera con una moneda y un banco central comunes.

${ }^{7}$ Sobre este tema, véase J. DARGin, "Qatar's Natural Gas: The Foreign Policy Driver”, Middle East Policy, 14 (2007), núm. 3, pp. 136-142.

${ }^{8}$ Una de esas posiciones puede encontrarse en M. Kamrava, "Royal Factionalism and Political Liberalization in Qatar", The Middle East Journal, 63 (2009), p. 403. 
del canal de televisión Al Jazeera en 1996 y la posterior insistencia en mantener abierta esa televisora con todo y su línea editorial, a pesar de las presiones externas que pugnan por su cierre o reforma. La segunda, otorgar asilo a diversos opositores políticos de países vecinos, y la tercera, el apoyo a ciertas organizaciones islamistas tenidas por terroristas en los países que han servido como aliados de Catar en distintas coyunturas.

Después del intento fallido de formar un canal de televisión satelital en el mundo árabe con sede en Arabia Saudita por parte de inversionistas y antiguos periodistas de la famosa British Broadcasting Corporation (BBC), el gobierno de Hamad Al Thani, que había llegado al poder hacía apenas unos meses, abrió las puertas a los promotores de tal iniciativa y de esa manera surgió Al Jazeera en $1996 .{ }^{9}$ Comenzando con transmisiones en lengua árabe solamente, la televisora obtuvo fama internacional por su cobertura de la invasión a Afganistán por parte de una coalición internacional liderada por Estados Unidos en 2001. Poco después, el cuerpo directivo de la empresa anunció planes de expansión que incluyeron la apertura de un canal en idioma inglés en 2006. Desde su fundación a mediados de los años noventa, Al Jazeera ha sido elemento primordial de las relaciones exteriores de Catar.

La línea editorial de Al Jazeera, que incluye críticas constantes a gobiernos dentro y fuera del mundo árabe, ha provocado tensiones diplomáticas a Catar. Por ejemplo, como protesta por ciertos contenidos mostrados en ese canal, Arabia Saudita retiró a su embajador en Catar entre 2005 y 2009. La tensión en la relación entre Doha y Riad durante esos años sirvió de precedente para el retiro del embajador saudí en Catar, en 2014, lo cual fue secundado por los gobiernos de Bahréin y Emiratos Árabes Unidos. A pesar de que al cabo de unos cuantos meses ese diferendo parecía resuelto, las

${ }^{9}$ Sobre la fundación de Al Jazeera, véase H. Miles, Al-Jazeera: The Inside Story of the Arab News Channel that is Challenging the West, Nueva York, Grove Press, 2005, pp. 41-43. 
tensiones se presentaron de nueva cuenta tres años después. En esa ocasión, esos mismos países y otros, como Egipto y Jordania, no sólo retiraron a sus embajadores en Doha, sino que impusieron un bloqueo a Catar, el cual estuvo vigente hasta mediados del 2018. En esos eventos, la televisora ha estado en el centro de las disputas.

La cobertura de Al Jazeera ha provocado también tensiones entre Catar y su principal aliado militar, Estados Unidos. Estas tensiones alcanzaron el punto crítico durante la administración de George W. Bush, a causa de las transmisiones de la televisora durante las invasiones a Afganistán, en 2001, e Irak, en 2003. Un punto de controversia fue la transmisión de los mensajes de Al-Qaeda mediante grabaciones hechas por el líder de esa organización, Osama Bin Laden. Como parte de ese diferendo, Bush llegó a denominar a Al Jazeera como "la televisora de los terroristas". En noviembre de 2005, el periódico británico The Daily Mirror publicó una nota en que se aseguraba que en una reunión con el Primer Ministro Británico, Tony Blair, el entonces presidente estadounidense propuso bombardear las oficinas centrales de la televisora en Doha. ${ }^{10}$ Aunque a los pocos días la Casa Blanca negó el alegato, ${ }^{11}$ la posibilidad de que esa conversación haya tenido lugar se suma a una serie de tensiones entre Catar y su principal garante de seguridad militar, Estados Unidos. A pesar de la recurrencia de incidentes diplomáticos como éstos, el gobierno de Catar no ha cedido a las presiones por cerrar el canal de televisión; por el contrario, ha propugnado por mantenerla operando, conservar su línea editorial intacta y dar paso firme para aumentar su audiencia.

Además de la reticencia por ceder a la presión de otros actores internacionales por censurar $\mathrm{Al}$ Jazeera, Catar ha lan-

${ }^{10}$ La nota puede encontrarse en K. Maguire y A. Lines, "Exclusive: Bush Plot to Bomb His Arab Ally", The Daily Mirror, 22 de noviembre de 2005, en http://www.mirror.co.uk/news/, consultado el 15.V.2017.

${ }^{11}$ Véase "Bush Al-Jazeera «Plot» Dismissed", BBC News, 22 de noviembre de 2005, en http://news.bbc.co.uk/2/hi/uk_news/politics/4459296. stm, consultado el 18.V.2017. 
zado otras iniciativas intrigantes que contradicen también las líneas generales de política exterior desde su independencia. Otra de esas iniciativas es otorgar asilo a diversos opositores políticos de países vecinos. Por ejemplo, Catar ha otorgado asilo a líderes políticos palestinos, como Azmi Bishara, quien como miembro de la Knesset fue un fuerte crítico de las políticas segregacionistas y expansionistas del Estado de Israel. De igual forma desde 2012, Khaled Meshaal, líder de Hamas, grupo de resistencia islámica en Palestina, reside de forma permanente en Doha. Finalmente, el jeque egipcio Yusuf alQaradawi radica desde hace varias décadas en Doha, desde donde transmite sus enseñanzas mediante un programa en $\mathrm{Al}$ Jazeera, el cual cuenta una audiencia de casi 25 millones de televidentes. ${ }^{12}$ Por sus vínculos con la Hermandad Musulmana, sobre todo en su juventud, y sus perspectivas críticas frente a distintos gobiernos en su natal Egipto, al-Qaradawi es considerado como un opositor político en su país de origen.

Otorgar asilo a estos y otros personajes no sólo ha provocado tensiones entre Catar y sus compañeros del CCG, sino también la ira de gobiernos de países vecinos, especialmente Egipto e Israel, cuyas capacidades militares exceden por mucho las de Catar. Uno de los temas que ha provocado el distanciamiento entre Catar y algunos de los países del CCG es la presencia de líderes de organizaciones como Hamás y la Hermandad Musulmana en Doha. Por ejemplo, el gobierno de Emiratos Árabes Unidos protestó ante la embajada de Catar en su país por declaraciones de al-Qaradawi, emitidas en uno de sus programas en 2014. ${ }^{13}$ Asimismo, una de las condiciones de los gobiernos vecinos para levantar el bloqueo impuesto a Catar a mediados de 2017 es la expulsión de algunos de esos opositores políticos, lo cual ha sido rechazado por Doha. A pesar de que esa política ha provocado

${ }^{12}$ A. B. Soage, "Shaykh Yusuf al-Qaradawi: Portrait of a Leading Islamic Cleric", Middle East Review of International Affairs, 12 (2008), p. 51.

13 "UAE Summons Qatari Ambassador over Cleric's Comments", Arabian Business.Com, 2 de febrero de 2014 (extraído de Factiva). 
problemas diplomáticos a Catar, el gobierno de este país se ha negado a expulsar a esos asilados políticos y ha mantenido las puertas abiertas a opositores de regímenes de países vecinos.

Una tercera iniciativa que ha provocado tensiones diplomáticas al gobierno de Catar es, en fin, el apoyo a ciertas organizaciones consideradas como terroristas por gobiernos de países vecinos y de países aliados. Por ejemplo, en distintos momentos durante las últimas décadas, el gobierno de Catar ha sido uno de los principales benefactores de Hamás y Hezbolá, dos organizaciones consideradas como terroristas por los gobiernos de Estados Unidos y algunos de la Unión Europea. Por lo demás, una coyuntura importante para la diplomacia catarí ocurrió cuando el gobierno de la Hermandad Musulmana en Egipto, que había sido apoyado por Catar, fue derrocado por un golpe de Estado en 2012. Después del golpe, el gobierno de Catar continuó apoyando a la Hermandad Musulmana en contra de los deseos de algunos gobiernos de la zona, lo cual desató la ira del gobierno egipcio del general Abdel Fattah el-Sisi.

Brindar apoyo a tales organizaciones también ha tenido consecuencias negativas para la diplomacia catarí, en especial por lo que toca a sus relaciones con países de la región y con aliados internacionales. Por ejemplo, una de las razones para imponer, en 2007, un bloqueo sobre Catar fue el alegato de que el gobierno de este país patrocinaba el terrorismo internacional. Aún más: el Presidente estadounidense Donald Trump, mediante su cuenta de Twitter, celebró el bloqueo contra Catar como un logro de su administración en la lucha contra el terrorismo. Esto debe sumarse a alegatos según los cuales Estados Unidos debería retirar su apoyo a Catar a causa de su cercanía con organizaciones terroristas, ${ }^{14}$ lo cual, si llegara a concretarse, sería un duro

${ }^{14}$ La presión de ciertos miembros del equipo de seguridad de Barack Obama por retirar la presencia militar estadounidense en Catar fue reportada en el periódico The Wall Street Journal. A este repsecto, véase J. Solo- 
golpe para la estrategia de la seguridad catarí, ya que perdería a su principal aliado militar. Con todo, el gobierno de Catar ha mantenido su política para con tales organizaciones, la cual incluye mantener canales de comunicación abiertos y apoyarlas abiertamente en ciertas coyunturas. Al mismo tiempo, su gobierno ha reiterado su llamado a revisar el término terrorista para evitar denominar como tal a organizaciones cuya agenda es más bien la resistencia en contra del despojo. ${ }^{15}$

En suma, la protección irrestricta de Al Jazeera, el otorgamiento de asilo a opositores políticos de países vecinos y el apoyo a ciertas organizaciones consideradas como terroristas por los gobiernos de países aliados constituyen los rasgos intrigantes de las relaciones exteriores cataríes. A pesar de las tensiones y conflictos diplomáticos que estas iniciativas han suscitado, el gobierno de Catar ha mantenido su posición con respecto de esos temas. Por esta razón, distintas perspectivas han indagado sobre las razones de tal obstinación, según se verá líneas abajo.

\section{Perspectivas de las relaciones exteriores de Catar}

Una de las principales explicaciones acerca de las relaciones exteriores de Catar privilegia la existencia de una estrategia del gobierno catarí para posicionar el país como una marca comercial en el ámbito internacional con el propósito de mejorar su entorno de seguridad y sustentar los negocios de la élite nacional. Según esta perspectiva, tal estrategia com-

\footnotetext{
mon y N. Malas, "Qatar's Ties to Militants Strain Alliance”, The Wall Street Journal, 23 de febrero 2015, en https://www.wsj.com/articles, consultado el 16.VIII.2017.

15 Un clamor de este tipo fue pronunciado por el emir de Catar, Tamim Al Thani, en la víspera de su primera visita a Estados Unidos como jefe de Estado. Al respecto, véase T. bin H. Al-Thani, "Qatar's Message to Obama", The New York Times, 24 de febrero de 2015, en https:/ /www.nytimes.com/2015, consultado el 18.VIII.2017.
} 
porta ser sede de actos internaciones ${ }^{16}$ e incrementar el protagonismo regional a partir de la mediación en conflictos. ${ }^{17}$ En este sentido, al analizar la política exterior catarí durante los primeros años del gobierno de Hamad Al Thani, J. E. Peterson comentó que

dos factores han influido la integración de Catar al sistema internacional. El primero son los retos y problemas que enfrentan los Estados pequeños y el segundo es la respuesta específica de Catar, la cual ha consistido en promocionar el Estado como una marca para alcanzar seguridad. ${ }^{18}$

El argumento de Peterson parecería desfasado después de la participación de Catar en los conflictos en países cercanos durante los conflictos que siguieron a la ola de protestas populares en el Medio Oriente, conocidas como Primavera Árabe. En esa circunstancia, la política exterior de Catar privilegió la intervención en varios de esos conflictos en franco apoyo de alguna de las partes. Sin embargo, ha habido quienes han intentado actualizar el argumento sobre posicionamiento de marca para incluir los más recientes desarrollos en ese razonamiento. Por ejemplo, para Sultan Barakat, la sustitución de mediación por intervención en la postura de Catar para con algunos conflictos en otros países no representa una trasformación en la estrategia de su gobierno, sino una reacción ante las demandas de definición por parte de la po-

${ }^{16}$ En décadas recientes, Catar ha sido sede de magnos actos internacionales, como el inicio de las negociaciones de la Ronda de Doha de la Organización Mundial del Comercio, los Juegos Asiáticos de 2006 y, próximamente, la Copa Mundial de Futbol de la FIFA.

${ }^{17}$ Sobre la mediación de Catar en conflictos en otros países, véase M. Kamrava, "Mediation and Qatari Foreign Policy", The Middle East Journal, 65 (2011), pp. 539-556.

18 "Qatar and the World: Branding for a Micro-State", The Middle East Journal, 60 (2006), p. 732. Salvo indicación en contrario, tanto ésta como las que siguen son traducciones mías. 
larización política en el mundo árabe a inicios del siglo XXI. ${ }^{19}$ De esta forma, la posición de Catar ante la Primavera Árabe se considera parte de una estrategia de posicionamiento de marca que incluye nuevos elementos, como la intervención activa en contra de regímenes represores y el apoyo a las iniciativas de países occidentales en el Medio Oriente. ${ }^{20}$

Privilegiar la estrategia de posicionamiento de marca como móvil fundamental de la actividad diplomática catarí ha aportado, sin duda, una perspectiva interesante para entender distintos aspectos de la diplomacia de este país del Golfo. Sin embargo, esta perspectiva no permite entender esos rasgos de las relaciones exteriores de Catar explicados en el apartado anterior, los cuales hacen de la actividad diplomática de este país un asunto intrigante. Las iniciativas antes mencionadas, en particular, quedan fuera del ámbito explicativo de esta perspectiva o incluso contradicen los supuestos de las que ésta parte. Por esta razón, la primacía de la estrategia de posicionamiento de marca como móvil fundamental del activismo regional catarí es insuficiente para entender el carácter impredecible y poco ortodoxo de la diplomacia de ese país.

Echar un vistazo a esas iniciativas ayudará a entender el porqué de la consideración del párrafo anterior. Para empezar, si bien la cadena televisiva Al Jazeera es un elemento importante para poner a Catar en el mapa, las razones por las que Al Jazeera ha dado a conocer a éste no son siempre las más adecuadas por lo que toca a la publicidad, sobre todo cuando su nombre se relacionó con el discurso sobre el terrorismo internacional. Por tanto, como estrategia de mercadeo, Al Jazeera no es la mejor opción. Algo semejante ocurre con los perseguidos políticos que encuentran asilo en Catar. Si el objetivo es darse a conocer, otorgar asilo a los opositores

${ }^{19}$ Qatari Mediation: Between Ambition and Achievement, Washington, DC, Brookings Institution, 2014. (Brookings Doha Center Analysis Paper, núm. 12).

${ }^{20}$ Para una afirmación similar, véase M. Gray, Qatar: Politics and the Challenges of Development, Boulder, Lynne-Rienner, 2013, p. 194. 
políticos no necesariamente da a conocer a Catar de la manera en que su gobierno esperaría. Por último, el apoyo a organizaciones consideradas como terroristas por aliados y vecinos también podrá dar a conocer a Catar, pero no por buenas razones. De hecho, los opositores políticos de su gobierno podrían utilizar los antecedentes de supuesto apoyo a grupos terroristas para dañar la imagen del país en Occidente y, de esa manera, socavar la estrategia de seguridad y las ambiciones de negocios de la élite catarí.

En pocas palabras, equiparar las relaciones exteriores de Catar con una mera estrategia de mercadeo deja de lado iniciativas muy importantes que han estado presentes en la relación del gobierno de ese país con el mundo exterior. A pesar de que las relaciones exteriores de Catar incluyan un intento por posicionar al Estado como una marca internacional, hay sin duda dinámicas que quedan fuera de esa lógica. Al enfrentarse a ellas, los proponentes de esta perspectiva simplemente las ignoran o las hacen a un lado. En lugar de ello, en este artículo se sugiere buscar en otro lugar para encontrar explicaciones de tales iniciativas.

Otra explicación recurrente sobre las relaciones exteriores de Catar apunta hacia una estrategia de seguridad basada en la formación del mayor número posible de alianzas. Más que una simple estrategia de mercadeo, esta perspectiva ve las relaciones exteriores de Catar como un intento constante por incrementar su relevancia en la agenda de otros actores regionales e internacionales mediante lazos de amistad, creación de vínculos estratégicos y construcción de agendas comunes. De esta manera, la seguridad de Catar, según se afirma, estaría garantizada, ya que cada vez serían más los actores cuyos intereses estarían estrechamente vinculados a la permanencia de Catar como un Estado independiente. La política exterior de Catar se reduce así a un intento incesante por garantizar la viabilidad como Estado independiente mediante una estrategia de formación de alianzas múltiples.

Usando el lenguaje de las apuestas, hay quienes han denominado cobertura la estrategia para ganar el mayor número 
de aliados posible. De esta manera, muchas de las iniciativas del gobierno de Catar en el exterior desde 1995 deberían entenderse como un esfuerzo por lograr esa cobertura. A propósito, Mehran Kamrava ha sostenido que

la estrategia de cobertura supone hacer una apuesta grande en un sentido, como las alianzas militares y de seguridad, por ejemplo, y hacer una serie de apuestas pequeñas en otro sentido, como con amigos de los que se puede prescindir, si es necesario. Para Catar, esto ha significado buscar protección por parte de Estados Unidos y, al mismo tiempo, mantener relaciones cordiales con Irán y Hamás. Las relaciones de Catar con Estados Unidos e Irán en particular, así como con Israel y Hamás, son emblemáticas de su estrategia de cobertura. ${ }^{21}$

Según esta perspectiva, para garantizar su viabilidad como Estado independiente, el gobierno de Catar ha decidido diversificar su sistema de alianzas, involucrándose con todo tipo de actores.

Análisis más acotados sobre ciertos aspectos de la política exterior catarí han retomado esta perspectiva. Por ejemplo, en un análisis sobre la relación de Catar con Israel desde mediados de los años noventa, Uzi Rabi caracterizó la política de Doha para con el Estado judío como parte de una "intrincada e independiente política regional que implicó iniciar y mejorar relaciones con la mayoría de sus vecinos". ${ }^{22}$ De forma similar, Kristian Coates Ulrichsen, al analizar la posición del gobierno de Catar frente a la ola de protestas conocida como la Primavera Árabe, apuntó a la intención de crear vínculos estratégicos con los regímenes posrevolucionarios, especialmente con aquellos de corte islamista, como uno de los determinantes de esa posición. ${ }^{23}$ Así, uno de los imperativos p. 8 .

${ }^{21}$ Qatar: Small State, Big Politics, Ithaca, Cornell University Press, 2013,

22 "Qatar's Relations with Israel: Challenging Arab and Gulf Norms", The Middle East Journal, 63 (2009), p. 447.

${ }^{23}$ Qatar and the Arab Spring, Londres, Hurst \& Co Publishers, 2014. 
que ha guiado la posición del gobierno de Catar durante el actual período de inestabilidad regional fue, según este recuento, la intención de forjar alianzas con el objetivo de mejorar su entorno de seguridad.

Sin duda alguna, la preocupación de formar alianzas mediante estrategias de cobertura ha aportado perspectivas interesantes para entender distintos aspectos de las relaciones exteriores de Catar. De entre éstas, descuella el establecimiento de vínculos con un conjunto de actores, cuya definición política supone intereses contradictorios y, en ocasiones, conflictivos. Por ejemplo, mientras mantenía una oficina de negocios de Israel en Doha, el gobierno de Catar incrementaba su apoyo a grupos que se encuentran en franco conflicto con el Estado judío, como Hamás y Hezbolá. Sin embargo, lo plausible de esta explicación, es decir los elementos que quedan fuera de su ámbito explicativo, nos invita a seguir indagando para tratar de entender las relaciones exteriores de Catar desde mediados de los años noventa.

$\mathrm{Al}$ igual que las supuestas estrategias de comercialización y posicionamiento de marca, la formación de alianzas múltiples es también insuficiente para entender los tres componentes de las relaciones exteriores de Catar, ya señalados al inicio de este artículo. En primer lugar, la fundación y el continuo funcionamiento de Al Jazeera ha ayudado a muchas cosas, excepto a formar alianzas con actores externos. Por el contrario, desde sus inicios $\mathrm{Al}$ Jazeera ha causado problemas diplomáticos al gobierno de Catar dentro y fuera del mundo árabe. Quizá los problemas más importantes han sido con sus compañeros dentro del CCG y con su principal aliado militar, el gobierno de Estados Unidos. Sin embargo, también llaman la atención los problemas con la Autoridad Nacional Palestina (ANP), que surgieron después de que en 2011 la televisora publicara un conjunto de documentos en que se daba a conocer la voluntad de la ANP para hacer concesiones comprometedoras al gobierno de Israel, como la cesión de Jerusalén. A los pocos días, hubo en la ciudad de Ramala, en Cisjordania, manifestaciones de parte de grupos 
de apoyo de la ANP en contra de Al Jazeera y del gobierno de Catar. ${ }^{24}$

De igual manera, el apoyo a organizaciones consideradas como terroristas y el otorgamiento de asilo a perseguidos u opositores políticos de países vecinos pueden asegurar al gobierno de Catar distintos aliados, sobre todo entre grupos que tienen influencia en la región del Medio Oriente. Sin embargo, esas iniciativas le han causado conflictos con actores muy poderosos en la región, en especial con el Estado de Israel, que cuenta con el décimo ejército más poderoso del mundo; con Egipto, cuya capacidad militar supera a la de cualquier otro país árabe, y Arabia Saudita, el país más poderoso del CCG. Más aún: estas iniciativas le han ocasionado tensiones con aquellos poderes extrarregionales que podrían garantizar la seguridad de Catar, principalmente Estados Unidos, pero también Gran Bretaña y Francia. Estas iniciativas contradicen la búsqueda de seguridad, considerada como el imperativo detrás de la estrategia de formación de alianzas múltiples. Entender las relaciones exteriores de Catar, por tanto, implica establecer marcos conceptuales más allá de estrategias de mercadeo y de formación de alianzas múltiples, según lo intento líneas abajo.

\section{LA ESFERA PÚBLICA ÁRABE Y LAS RELAGIONES EXTERIORES DE LOS ESTADOS DE LA REGIÓN}

Para reiterar el argumento aquí expuesto, este artículo se concentra en el influjo de la esfera pública árabe en las relaciones exteriores de los Estados del Medio Oriente. Mediante el análisis de la proyección internacional de Catar, es posible observar la forma en que las estructuras estatales, por medio

${ }^{24}$ Sobre estos eventos, véase H. Sher wood, "Palestine Papers Provoke Anger on Streets of West Bank and Gaza”, The Guardian, 25 de enero de 2011, en https://www.theguardian.com/world/2011, consultado el 3.VI.2017. 
de sus relaciones exteriores, interactúan con las tendencias en una esfera pública trasnacional compuesta por plataformas tecnológicas de comunicación. En Catar, el influjo de la esfera pública árabe en el proceso de toma de decisiones se manifiesta en el lanzamiento de iniciativas con gran valor simbólico, como rechazar las presiones para cerrar o cambiar la línea editorial de la televisora Al Jazeera, ofrecer apoyo constante a organizaciones consideradas como terroristas por parte de sus aliados internacionales y otorgar asilo a opositores políticos de varios Estados vecinos. Por lo demás, la manifestación concreta de la esfera pública árabe en las relaciones exteriores de Catar se debe a características propias.

Toda vez que la interacción con las tendencias en la esfera pública árabe por parte de su líder es un componente fundamental de la conducción de las relaciones exteriores de Catar, es necesario aclarar qué significa el término esfera pública. El concepto de esfera pública pasó por un proceso de renovación y auge en las Ciencias Sociales a partir de las aportaciones del filósofo alemán Jürgen Habermas. En su libro The Structural Transformation of the Public Sphere, ${ }^{25}$ éste acuñó el término para designar ese espacio de encuentro entre individuos privados y autoridades de gobierno en el que se intercambiaban puntos de vista sobre asuntos públicos a partir de reuniones en cafés, publicaciones en prensa escrita, difusión de piezas de arte. La apertura de este espacio de discusión crítica y racional en la Europa de finales del siglo XviI dio lugar, al cabo de siglo y medio, al surgimiento de sociedades liberales y burguesas en las que el poder soberano enfrentaba límites inexistentes en las sociedades otrora feudales. Tras esta estela, la esfera pública es, pues, una suerte de espacio imaginario que provee las bases para la expansión de ideas por medio de innovaciones tecnológicas. ${ }^{26}$

25 Trad. de T. Burger, Cambridge, miт Press, 1991.

${ }^{26}$ El concepto de esfera pública planteado por Habermas ha sido sometido a críticas diversas. A este respecto, véase C. CAlHoun (ed.), Habermas and the Public Sphere, Cambridge, MIT Press, 1992. 
En el Medio Oriente, como en gran parte del mundo poscolonial, ${ }^{27}$ surgió a finales del siglo xıx un movimiento literario llamado Resurgimiento Árabe (al-Nahda al-Arabiyya), el cual habría de sentar las bases para el subsecuente surgimiento de una esfera pública árabe y de sentimientos de identificación colectiva como el nacionalismo árabe. En este sentido, en su trabajo sobre los orígenes de la identidad árabe moderna, Stephen Sheehi demuestra la importancia de la creación de clubes literarios en las principales capitales del mundo araboparlante durante la segunda mitad del siglo xix para el subsiguiente desarrollo de una esfera pública urbana en el Medio Oriente, conformada principalmente por varones letrados provenientes de las élites locales. ${ }^{28}$ La esfera pública surgida de esos clubes fue el antecedente de proyectos políticos que surgieron en el mundo árabe durante el colonialismo europeo de las primeras décadas del siglo xx. Por ejemplo, la revuelta en contra del Imperio otomano liderada por el Jerife de la Meca, Hussein bin Ali, e instigada por el gobierno británico, se nutrió de la esfera pública árabe, y fue después conocida como la Gran Revuelta Árabe. ${ }^{29}$ Al igual que en Europa, después del siglo xviI, surgió en el Medio Oriente una esfera pública durante el siglo xix que habría de influir en procesos posteriores en esa región.

En décadas más recientes, el cambio tecnológico ha influido en el crecimiento y la transformación de la esfera pública en el mundo árabe. A mediados del siglo pasado, la

${ }^{27}$ Para efectos de este trabajo, el término poscolonial se refiere principalmente a aquellas regiones de Asia y África que para mediados del siglo xx habían alcanzado su independencia después de haber formado parte de imperios europeos.

28 Foundations of Modern Arab Identity, Gainesville, University Press of Florida, 2004.

${ }^{29}$ Esto no excluye las dimensiones locales de la revuelta, que incluían aspectos ajenos al imaginario árabe y tenían más que ver con la relación de los campesinos con la Sublime Puerta y el desencanto por el incremento de los impuestos. Sobre este tema, véase M. Provence, The Great Syrian Revolt and the Rise of Arab Nationalism, Austin, University of Texas Press, 2005 . 
llegada de la comunicación por ondas radiofónicas expandió el alcance de la esfera pública, incluidas zonas rurales, distribuyendo información a personas iletradas que habían sido excluidas de los anteriores clubes literarios. ${ }^{30}$ Fue de esta manera como el gobierno egipcio del presidente Gamal Abdel Nasser creó, en 1953, la radio La Voz de los Árabes (Sawt al-'Arab), la cual, a más de difundir el programa de gobierno de Nasser, expandió esa esfera pública para incluir sectores previamente excluidos. Más recientemente, el surgimiento de la televisión satelital y de las redes sociales de Internet han hecho una aportación muy importante por lo que a esto atañe. Por ejemplo, Marc Lynch ha apuntado que los talk show de Al Jazeera, que permiten la interpelación del público con los expositores, han creado una esfera pública alternativa a las televisoras dominadas por el Estado en el mundo árabe. ${ }^{31}$

Los Estados árabes han estado íntimamente ligados a la esfera pública árabe desde el surgimiento del sistema regional de Estados independientes en el Medio Oriente en las primeras décadas del siglo xx. Durante la época poscolonial, la promoción de formas de identificación colectiva que se concentraba en imaginarios más localizados (qawmiyya alwataniyya) ${ }^{32}$ entró en conflicto con la identidad árabe (qawmiyy a al-arabiyya), lo que llevó a muchos a considerar las fronteras de los nuevos Estados-nación como divisiones ficticias que obedecían a las pretensiones de dominación de los poderes

${ }^{30}$ La televisión satelital como plataforma para el desarrollo de la esfera pública árabe es el argumento principal de la siguiente obra: M. LYNCH, Voices of the New Arab Public: Iraq, Al Jazeera, and Middle East Politics Today, Nueva York, Columbia University Press, 2007, pp. 60-62. Para un argumento semejante en cuanto a la esfera pública musulmana, véase D.F. Eickelman y J. W. Anderson, New Media in the Muslim World: The Emerging Public Sphere, Bloomington-Indianapolis, Indiana University Press, 1999.

31 Op. cit., p. 4.

32 En varias ocasiones, esas identidades colectivas más localizadas coincidían con los límites de las antiguas provincias otomanas en la región del Levante. 
otrora coloniales europeos. Por ejemplo, un elemento central de la ideología del partido Baath, que ostentó el poder en Irak por más de treinta años y en Siria desde 1963, fue la abolición de las fronteras nacionales, ya que las consideraba una imposición externa. ${ }^{33}$ Para algunos, una división territorial que verdaderamente reflejara la identidad local debería abarcar toda la nación árabe, incluida la Palestina histórica. ${ }^{34}$ Entre esas pretensiones, el proyecto más acabado fue la creación, en 1958, de la República Árabe Unida entre Siria y Egipto, que se abandonó con el tiempo. ${ }^{35}$ Por lo demás, todas estas demandas y proyectos son ejemplos específicos de los alcances de la esfera pública árabe en el Medio Oriente durante la época poscolonial.

Durante la segunda mitad del siglo xx, la esfera pública árabe continuó siendo muy relevante para los Estados árabes y para sus relaciones mutuas. En este sentido, Michael Barnett ha calificado las relaciones internacionales en la región como un diálogo entre los distintos Estados árabes a partir de tres temas. ${ }^{36} \mathrm{El}$ primero es la posición ante los poderes coloniales, primero europeo y luego estadounidense. La segunda es la posición ante los procesos de integración y de creación de una estructura estatal que refleje la identidad árabe. La tercera, en fin, es la posición ante el Estado de Israel, que ha sido considerado como un enclave colonial en el mundo árabe desde su fundación en 1948. Según esta perspectiva, la posición de los distintos Estados árabes frente a estos tres temas constituye los diálogos en la política interárabe, lo cual demuestra la relevancia de la esfera pública árabe

33 D. S. Patel, "Repartitioning the Sykes-Picot Middle East? Debunking Three Myths", Middle East Brief, 2016, núm. 103, p. 5.

34 Sobre este tema, véase R. KHALidi, "The Origins of Arab Nationalism: Introduction”, en R. Khalidi et al. (eds.), The Origins of Arab Nationalism, Nueva York, Columbia University Press, 1991, pp. VII-XIX.

${ }^{35} \mathrm{Al}$ respecto, véase, E. PoDeH, The Decline of Arab Unity: The Rise and Fall of the United Arab Republic, Sussex, Academic Press, 1999.

36 Dialogues in Arab Politics: Negotiations in Regional Order, Nueva York, Columbia University Press, 1998. 
para los gobiernos de los Estados árabes durante la segunda mitad del siglo pasado. ${ }^{37}$

El declive de los proyectos lanzados en nombre del nacionalismo árabe y la aparente resistencia de las identidades nacionales no ha minado la importancia de la esfera pública árabe en la política regional. La muestra más clara de ello es la ola de protestas que se suscitó en casi todos los países de la región después de la autoinmolación de un vendedor ambulante en Túnez, en el 2011. La llamada Primavera Árabe demostró que los procesos de comunicación trasnacionales en esa región tienen consecuencias importantes en procesos políticos regionales en el Medio Oriente. Esto se debe a diversas razones. La primera de ellas es la articulación de demandas políticas a partir de un marco trasnacional, lo cual provoca, por ejemplo, que hechos ocurridos en Palestina resuenen con fuerza en regiones distantes del norte de África y del Golfo. La segunda razón es que los Estados árabes tienen la posibilidad de incrementar su influencia regional y su posición interna, si mejora su reputación en la esfera pública árabe. Por estas razones, como Lynch ha señalado, al analizar los aspectos de identidad en la política exterior del reino de Jordania, los Estados árabes están interesados en atender las tendencias en la esfera pública árabe mediante actos con gran valor simbólico y, de esa manera, mejorar su posición y reputación en el entorno regional. ${ }^{38}$

A pesar de su breve historia como Estado-nación, Catar no es distinto del resto de los Estados árabes con respecto de su posicionamiento y relación con la esfera pública árabe. Como el resto de sus homólogos en la región, el gobierno de Catar tiene como una de sus consideraciones importantes atender los desarrollos en esa esfera pública a partir de sus relaciones exteriores. Así, además de prestar atención a los imperativos

37 Idem.

38 "Jordan's Identity and Interests", en S. Telhami y M. Barnett (eds.), Identity and Foreign Policy in the Middle East, Ithaca, Cornell University Press, 2002. 
de seguridad estatal y a los retos para la supervivencia del régimen, las relaciones exteriores de Catar están influidas por una necesidad de atender los desarrollos en la esfera pública árabe. Sin embargo, ésta no es una respuesta automática a los estímulos de una fuerza estructural que excluye posibilidades de acción distintas. Por el contrario, el involucramiento activo con la esfera pública árabe otorga a las relaciones exteriores cataríes su carácter distintivo e impredecible. Ese activismo internacional contrasta no solamente con el patrón en las relaciones exteriores de Catar durante los primeros veinticinco años de su vida independiente, sino también con la diplomacia del resto de los Estados de la región. De las razones de este activismo distintivo en la esfera pública árabe doy cuenta a continuación.

La primera de esas razones es el inmenso flujo de capital a Catar a partir del incremento sin precedentes del precio internacional del petróleo entre los años 2002 y 2008. Ulrichsen, en su análisis sobre el papel de los Estados árabes del Golfo en la economía global, ha apuntado el masivo flujo de capital, principalmente a Catar y los Emiratos Árabes Unidos, como una de las razones principales por detrás del creciente activismo regional de esos Estados y de su advenimiento como actores de renombre internacional, ${ }^{39}$ lo cual es particularmente cierto en el caso catarí. Por su pequeña población (de aproximadamente dos millones y medio de habitantes hacia 2016) y a sus grandes reservas de gas natural (una de las tres reservas nacionales más grandes del mundo), Catar amasó una fortuna inmensa durante ese período; así, pasó a ocupar el primer lugar en el índice del Fondo Monetario Internacional según el ingreso per capita de los países del mundo en 2010. El inmenso flujo de capital ha sido entonces el elemento material que ha permitido el surgimiento de esta política asertiva, cuyo objetivo es mejorar la posición y reputación de Catar en la esfera pública árabe.

39 The Gulf States in International Political Economy, Nueva York, Palgrave Macmillan, 2016, p. 103. 
El segundo factor es la centralización del proceso de toma de decisiones en la figura del Emir (primero Hamad y luego Tamim) y de sus más cercanos colaboradores. Por ejemplo, ha sido una persona muy influyente en ambos períodos la presidente de la Fundación Catar, Mozah bint Nasser alMissned, cuyo esposo es el Emir anterior. Esta centralización del proceso de toma de decisiones en una familia permite pensar que la forma como Catar ha conducido sus relaciones exteriores desde principios del siglo es también reflejo de las pretensiones personales del jefe de Estado en turno y de sus más cercanos colaboradores, quienes se han mantenido más o menos en sus puestos durante las últimas dos décadas. En otras palabras, uno de los factores que ha distinguido las relaciones exteriores de Catar de las del resto de los Estados árabes son los intereses de quienes dirigen los asuntos públicos en ese país.

La relativa falta de oposición política interna al régimen con respecto del resto de los países árabes y, en especial, los Estados árabes del Golfo, es un factor que habilita la participación de Catar en la esfera pública árabe a partir de sus relaciones exteriores. La presencia de un Estado fuerte, promotor de un sentido de autocontrol entre la población, habilita el involucramiento activo por parte del gobierno de Catar en la esfera pública árabe sin temor a repercusiones negativas para su estabilidad, lo cual no ocurre ni en Arabia Saudita, ni en los Emiratos Árabes Unidos, que tienen, por ejemplo, una oposición más organizada, la cual se puede fortalecer por el aumento de la influencia de grupos afines en países vecinos. Por ello, estos países han adoptado una postura reaccionaria en los años posteriores a la Primavera Árabe, mientras que Catar se ha guiado más por el deseo popular en la esfera pública árabe.

En resumen, la existencia de una esfera pública trasnacional en el mundo árabe ha sido uno de los principales rasgos de las relaciones internacionales en el Medio Oriente durante la época poscolonial. Los desarrollos y tendencias en esta esfera pública árabe han sido un aspecto por conside- 
rar de parte de los países de la región, sobre todo en la conducción de sus relaciones exteriores. En el caso catarí, la interacción con la esfera pública árabe ha sido más notoria desde mediados de los años noventa a causa de una serie de factores propios.

\section{LOS RASGOS INTRIGANTES DE LA DIPLOMACIA CATARÍ ANTE A LA ESFERA PÚBLICA ÁRABE}

El propósito de esta sección es analizar los rasgos intrigantes de las relaciones exteriores de Catar, utilizando las herramientas conceptuales explicadas líneas arriba. Para ello, en las páginas siguientes se sostiene que un factor importante subyacente en el diseño e implementación de esas iniciativas diplomáticas es el involucramiento del régimen actual en Doha en la esfera pública árabe. ${ }^{40}$ En este sentido, durante una entrevista de televisión en medio de la ofensiva militar del ejército israelí en la Franja de Gaza, en 2009, el entonces primer ministro catarí, Hamad bin Jaber Al Thani, declaró que

la posición en la calle árabe con respecto de este asunto es más firme que nuestra posición como gobiernos. Para algunos, este levantamiento popular está conformado por un conjunto de personas indeseables que no merecen atención alguna. Pero eso no es verdad. Se trata, por el contrario, de un levantamiento árabe. Por tanto, nosotros, como gobiernos, deberíamos expresar la opinión de la gente; los presidentes deberían expresar la opinión de su gente. Sólo así se lograría la estabilidad en la región. ${ }^{41}$

${ }^{40}$ Como ya se mencionó, el régimen actual comenzó con la llegada al poder de Hamad bin Jalifa Al Thani en 1995 y continuó con el ascenso de su hijo, Tamim bin Hamad Al Thani, en 2013.

${ }^{41}$ El texto completo de la entrevista se encuentra en "Qatari Premier Calls for «Clear-Cut» Stand on Israeli «Aggression»", BBC Monitoring Middle East, 13 de enero de 2009. 
Tomando esa declaración como punto de partida, se analizan a continuación las iniciativas contradictorias e impredecibles de la diplomacia catarí desde mediados de los años noventa.

Para reafirmar lo dicho líneas arriba, el primer rasgo intrigante de la política exterior catarí es el empecinamiento por mantener abierta la televisora $\mathrm{Al}$ Jazeera, a pesar de los conflictos diplomáticos que su línea editorial ha provocado al gobierno de ese país. Por ejemplo, durante las ofensivas militares lideradas por Estados Unidos en Afganistán (2001) e Irak (2003), el entonces presidente de Estados Unidos, George W. Bush, llamó públicamente a Al Jazeera la "televisora de los terroristas”. Asimismo, las recientes crisis diplomáticas entre Catar y sus compañeros del CGG en 2014 y 2017 han tenido como uno de sus componentes centrales el cierre de $\mathrm{Al}$ Jazeera. A pesar de ello, el gobierno de Catar ha perseverado en su empeño de mantener abierta la televisora y ha apoyado sus planes de expansión.

El empecinamiento de mantener abierta Al Jazeera y preservar su línea editorial intacta contradice la supuesta estrategia catarí de seguridad para mantener relaciones amistosas con la mayoría de actores posibles y, como tal, es parte de los intentos del gobierno catarí para mejorar su posición en la esfera pública árabe. Como Lynch ha notado en su Voices of the New Arab Public (antes citado), en un contexto en que las tecnologías de la comunicación han sido controladas fuertemente por los Estados, la aparición de Al Jazeera introduce un elemento innovador en la política del Medio Oriente, al aportar un elemento de cobertura ajena a los dictados de los gobiernos regionales y al brindar la oportunidad de que ciertas voces críticas lleguen a una audiencia más amplia. Esta percepción de apertura y solidaridad para con las voces críticas de la esfera pública árabe es fácilmente transmitida a su país sede, lo que promueve la imagen de Catar como promotor de las causas más sensibles en el mundo árabe. En pocas palabras, la presencia de $\mathrm{Al}$ Jazeera dota a Catar de una imagen de solidaridad y apoyo para con las luchas populares en 
la región, entre las que descuella la del pueblo palestino en contra de la ocupación.

El papel de Al Jazeera en la esfera pública árabe es sin duda un asunto importante para el gobierno de Catar. Por ejemplo, en una entrevista en inglés para el periódico británico The Financial Times, concedida en octubre del 2010, Hamad $\mathrm{Al}$ Thani explicó sus razones para fundar Al Jazeera, cuando dijo que estaba "convencido de que la gente en el Medio Oriente debe tener la oportunidad de expresar sus opiniones libremente, sobre todo en medio de los sucesos actuales en la región". Al ser cuestionado sobre los problemas que la televisora ha causado al gobierno de Catar, Hamad respondió:

Al Jazeera me ha provocado muchos problemas. En diversas ocasiones, muchos líderes árabes no han querido dirigirme la palabra siquiera. Pero después han entendido que no voy a cambiar mi opinión con respecto de la televisora. ${ }^{42}$

El hecho de que el Emir mismo esté consciente de la falta de popularidad de $\mathrm{Al}$ Jazeera entre muchos gobiernos y de su aceptación entre segmentos importantes de la población de países árabes es una muestra de la importancia de esa televisora para el gobierno de Catar. De esta manera, se puede entender la negativa del gobierno catarí de acceder a las demandas de gobiernos vecinos y aliados con respecto del cierre o reforma de Al Jazeera.

El segundo rasgo intrigante de las relaciones exteriores de Catar durante las últimas dos décadas es el acercamiento y, en diversas ocasiones, franco apoyo, a organizaciones consideradas como terroristas por parte de sus aliados más importantes, en especial Estados Unidos. En este sentido, se destaca el apoyo para la reconstrucción del sur de Líbano después de la ofensiva militar israelí de 2006, lo cual supuso un acercamien-

42 Véase el texto completo de la entrevista en M. Dickson y R. KHALAF, "Interview Transcript: Sheikh Hamad, Emir of Qatar", The Financial Times, 24 de octubre de 2010. 
to entre el gobierno catarí y Hezbolá, la cual suelen considerar como una organización terrorista los gobiernos de Estados Unidos, de la Unión Europea, entre otros. De igual manera, el gobierno de Catar ha apoyado activamente los esfuerzos de reconstrucción en la franja de Gaza, lo cual lo ha llevado a promover la posición de Hamás en los distintos procesos de negociación entre el gobierno israelí y su contraparte palestina, sobre todo después de crisis militares.

El acercamiento a esas organizaciones ha provocado malestar entre los aliados de Catar, principalmente Estados Unidos, quien es considerado su principal aliado militar. Por ejemplo, en 2009, el entonces senador y posterior secretario de Estado norteamericano, John Kerry, afirmó en una conferencia en el Instituto Brookings que Catar "no podía seguir siendo un aliado de Estados Unidos los lunes, mientras enviaba dinero a Hamas los martes" y, por tanto, Washington debería revisar su relación con ese país. ${ }^{43}$ Asimismo, el subcomité para el Medio Oriente y el norte de África del Senado de Estados Unidos envió en 2014 al Departamento de Estado una carta firmada por 24 legisladores en que exhortaba, haciendo referencia explícita a la relación entre Catar y Hamás, a sancionar a cualquier entidad o nación que siguiera apoyando a esa organización considerada como terrorista. ${ }^{44} \mathrm{~A}$ pesar de las tensiones que esta posición ha provocado en su relación con aliados importantes como Estados Unidos, el gobierno de Catar ha mantenido su apoyo y acercamiento con esas organizaciones.

Según la tesis de este artículo, la insistencia del gobierno de Catar en acercarse y apoyar a esas organizaciones tiene que ver con su búsqueda de posicionamiento en la esfera

${ }^{43}$ P. Goodenough, "Kerry in 2009: Qatar Can't Be American Ally on Monday that Sends Money to Hamas on Tuesday", CNS News, 1 de agosto de 2014, en https://www.cnsnews.com/news, consultado el 10.VII.2017.

${ }^{44} \mathrm{El}$ texto de la carta se puede encontrar en la página de Internet de la otrora congresista estadounidense Ileana Ros-Lehtinen. Véase https:/ / ros-lehtinen.house.gov/press-release/ros-lehtinen-deutch-poe-and-sherman-lead-bipartisan-letter-treasury-department-seeking. 
pública árabe. En otras palabras, el apoyo a estas organizaciones ha sido elemento fundamental en la estrategia del gobierno de Catar por mejorar su posición y aumentar su reputación en la esfera pública árabe. Esta estrategia implica un involucramiento directo en los esfuerzos de reconstrucción y alivio de los estragos dejados por las ofensivas militares israelíes en los países vecinos. Porque Hamás y Hezbolá son autoridades de facto en zonas asoladas por la guerra (el sur de Líbano y Gaza, respectivamente), cualquier tipo de apoyo o esfuerzo de reconstrucción en esas zonas implica una cercana relación con esas organizaciones que se tienen por terroristas en Occidente. De esta manera, las aspiraciones regionales del gobierno de Catar contradicen su estrategia de lograr una alianza sólida con los países occidentales, principalmente Estados Unidos.

La importancia para el gobierno de Catar de su activismo regional y, en consecuencia, de su relación con organizaciones consideradas como terroristas en ciertos países queda evidenciada por su decisión de retar el entendimiento generalizado en Occidente respecto del terrorismo islámico. Al ser consciente de las tensiones entre su posición ante ciertos asuntos regionales y sus alianzas internacionales, el gobierno de Catar busca suavizar esas tensiones mediante un cuestionamiento abierto al concepto de terrorismo enarbolado por Occidente y, específicamente, a la clasificación de ciertas organizaciones terroristas por parte de gobiernos aliados. Por ejemplo, una de las declaraciones recurrentes de la delegación de Catar ante la asamblea general de las Naciones Unidas se ha concentrado en la necesidad de "definir terrorismo y distinguirlo claramente de las luchas libradas por los pueblos en el ejercicio pleno de su legítimo derecho de defender su libertad y gozar de autodeterminación". ${ }^{45}$ El hecho de que el gobierno de Catar se lance decididamente en esta ba-

45 State of Qatar, "Statement before the 60th Session of the General Assembly of the United Nations", Nueva York, 19 de septiembre de 2005, p. 5 . 
talla conceptual en un foro tan importante como la onU demuestra el papel crucial de la relación con esas organizaciones para su diplomacia regional.

El tercer rasgo intrigante de las relaciones exteriores de Catar es el otorgamiento de asilo dentro de sus fronteras a perseguidos políticos de países vecinos. Un punto de fricción constante durante las crisis diplomáticas en las que Catar se ha visto envuelto ha sido el hecho de que distintos personajes notables de la oposición en distintos países vecinos residan en Doha. Por ejemplo, en la crisis diplomática de 2017, uno de los puntos ríspidos fue la presencia en Doha de miembros de la oposición egipcia, como el jeque Yusuf al-Qaradawi, y de otros personajes influyentes en el mundo árabe, como los palestinos Khaled Meshaal y Azmi Bishara, líder de Hamas y nacionalista árabe, respectivamente. A pesar de ello, el gobierno en Doha ha continuado con esa política y ha reiterado su convicción de abrir sus puertas a ese tipo de huéspedes. Por ejemplo, al ser cuestionado en una conferencia de prensa en 2015 sobre la posibilidad de que el entonces vocero de Hamas, Khaled Meshaal, haya abandonado su residencia en Doha, el entonces ministro de relaciones exteriores de Catar, Khaled Al-Attiyah, recalcó que "Meshaal es un huésped muy apreciado en Catar, donde vive tranquilamente con su familia". 46

Como los otros dos rasgos intrigantes de las relaciones exteriores de Catar, éste se explica a partir de los intentos del régimen por posicionarse en la esfera pública árabe. Asilar a distintos opositores políticos es un elemento fundamental en esa estrategia, ya que ayuda a fortalecer los vínculos entre el gobierno catarí y los distintos liderazgos a lo largo y ancho del mundo árabe. De esta manera, el gobierno de Catar se asegura de aumentar sus redes en el mundo árabe, principalmente con actores no estatales, a partir de la cual podrá sustentar sus iniciativas en la región. Un ejemplo de ello es la

46 "Qatari FM Denies Reports on Meshaal Departure from Doha", Kuwait News Agency, 12 de enero de 2015. 
formación del Doha Institute, un centro de investigación dedicado a temas sociales que ha convertido a Catar en un centro de debate en el mundo árabe y cuyo director es el palestino Azmi Bishara. Según un reportaje del periódico británico Financial Times, Bishara ayudó al gobierno de Catar a establecer vínculos con sectores de la oposición siria para articular una estrategia de derrocamiento del gobierno de Bashar Al-Assad. ${ }^{47}$

El gobierno de Catar está consciente del malestar que provoca entre muchos países vecinos otorgar asilo a personajes de la oposición en el mundo árabe. Sin embargo, no ha cesado en su decisión de hospedar en Doha a tales personajes. Por lo demás, el hecho de considerarlos "huéspedes distinguidos" demuestra que no es un asunto menor para el gobierno de Catar contar con esa presencia dentro de sus fronteras. Por el contrario, este gobierno reconoce las ventajas diplomáticas que implica contar con buenas relaciones con tales individuos. Por tanto, al igual que garantizar la operación de $\mathrm{Al}$ Jazeera y promover un acercamiento con organizaciones consideradas como terroristas por parte de sus aliados occidentales, otorgar asilo a perseguidos políticos en países vecinos es parte de los intentos del gobierno de Catar por mejorar y consolidar su posición en la esfera pública árabe.

\section{Conclusión}

Como primera consideración final es necesario señalar de manera clara el papel del marco conceptual expuesto anteriormente en el entendimiento general de las relaciones exteriores del Estado de Catar. En este sentido, la atención a las tendencias al interior de la esfera pública árabe corre en pa-

47 Véase el reportaje completo en R. Khalaf y A. Fielding-Smith, "How Qatar Seized Control of the Syrian Revolution", The Financial Times, 17 de mayo de 2013, en https://www.ft.com/content/, consultado el 27.VII.2017. 
ralelo de otros factores, como consideraciones de seguridad estatal y estabilidad del régimen, que se han identificado como imperativos tradicionales en las relaciones exteriores de los Estados. Por tanto, el enfoque aquí expuesto no contradice tales aseveraciones, sino que las complementa mediante la atención a un factor ignorado, es decir las tendencias en una esfera pública trasnacional. En otras palabras, el argumento de este artículo no desplaza marcos tradicionales para entender las relaciones exteriores de los Estados árabes; tan sólo invita, más bien, a dirigir la atención a la esfera pública árabe y a discutir la forma en que las tendencias en su interior interactúan con los imperativos de seguridad para producir iniciativas específicas en el ámbito diplomático.

Todo lo cual se relaciona con la segunda consideración final. Como quedó demostrado líneas arriba, el estudio de las relaciones exteriores de Catar hace aportaciones importantes al estudio de las relaciones exteriores de los Estados árabes, ya que provee pistas para indagar en la forma como las tendencias al interior de la esfera pública árabe influyen en la actividad diplomática de los Estados de la región. El estado de Catar presenta ciertas características distintivas con respecto del resto de los Estados de la región, como el alto nivel de renta derivado de la venta de hidrocarburos, principalmente gas natural, la concentración del proceso de toma de decisiones en un círculo cerrado y la relativa falta de oposición al régimen dentro de sus fronteras. Estos factores posibilitan el involucramiento del Estado catarí en la esfera pública árabe mediante el lanzamiento de iniciativas de gran valor simbólico. Analizar la forma en que ciertos desarrollos regionales influyen en la proyección regional de un Estado con características particulares es, pues, la principal aportación del estudio de las relaciones exteriores de Catar a los debates sobre política exterior en el Medio Oriente.

La última consideración, relacionada con el punto anterior, atañe al valor del marco aquí propuesto para elaborar análisis comparados sobre las relaciones exteriores de Estados dentro y fuera del Medio Oriente. En este sentido, 
investigaciones futuras podrían abrevar en las herramientas conceptuales presentadas en estas páginas para elaborar análisis comparados de las relaciones exteriores de los Estados árabes e indagar sobre las diferencias y similitudes. Asimismo, el énfasis en la esfera pública árabe provee el lenguaje para reflexionar sobre la forma en que otras esferas públicas trasnacionales influyen en las relaciones exteriores de Estados más allá del Medio Oriente. Una propuesta oportuna sería indagar sobre la importancia de una posible esfera pública trasnacional en las relaciones exteriores de los Estados latinoamericanos. De esta manera, el marco aquí propuesto daría lugar a futuras investigaciones dentro y fuera de la región del Medio Oriente.

Por lo demás, el marco aquí propuesto sirve también para indagar sobre la proyección internacional de Catar en los años por venir. En este sentido, cobra especial importancia la relación entre Catar y los países vecinos, algunos de los cuales impusieron un bloqueo a mediados del 2017, que está vigente aun después de más de un año. A la luz de la tendencia de la política exterior catarí desde 1995 y la obstinación de su gobierno por mejorar su posición en la esfera pública árabe, es muy probable que las tensiones con sus vecinos se mantengan. En tal caso, Catar buscará fortalecer sus relaciones con otros países de la región, principalmente Turquía e Irán. De la misma forma, es muy probable que sus compañeros del CGG, especialmente Arabia Saudita y los Emiratos Árabes Unidos, continúen rechazando el activismo regional de Catar y mantengan o incrementen incluso la presión para cambiar la línea de política exterior de Doha. En pocas palabras, las relaciones entre los Estados árabes del Golfo auguran un escenario de inestabilidad en los años por venir y este artículo provee herramientas conceptuales para interpretar los desarrollos políticos en una región crucial para el mundo. 


\section{REFERENCIAS BIBLIOGRÁFICAS}

Al-Thani, Tamim bin Hamad, "Qatar's Message to Obama", The New York Times, 24 de febrero de 2015, en https://www.nytimes.com/2015

Barakat, Sultan, Qatari Mediation: Between Ambition and Achievement, Washington, DC, Brookings Institution, 2014. (Brookings Doha Center Analysis Paper, núm. 12).

Barnetr, Michael, Dialogues in Arab Politics: Negotiations in Regional Order, Nueva York, Columbia University Press, 1998.

"Bush Al-Jazeera «Plot» Dismissed", BBC News, 22 de noviembre de 2005, en http://news.bbc.co.uk/2/hi/uk_news/politics/4459 296.stm

Calhoun, Craig (ed.), Habermas and the Public Sphere, Cambridge, The MIT Press, 1992.

Dargin, Justin, "Qatar's Natural Gas: The Foreign Policy Driver", Middle East Policy, 14 (2007), núm. 3, pp. 136-142.

Dickson, Martin y Roula KHalaf, "Interview Transcript: Sheikh Hamad, Emir of Qatar”, The Financial Times, 24 de octubre de 2010.

Eickelman, Dale F. y Jon W. Anderson, New Media in the Muslim World: The Emerging Public Sphere, Bloomington-Indianapolis, Indiana University Press, 1999.

Gause, Gregory, The International Relations of the Persian Gulf, Cambridge, University Press, 2010.

Goodenough, Patrick, "Kerry in 2009: Qatar Can't Be American Ally on Monday that Sends Money to Hamas on Tuesday", CNS News, 1 de agosto de 2014, en https://www.cnsnews.com/news Gray, Matthew, Qatar: Politics and the Challenges of Development, Boulder, Lynne-Rienner, 2013.

Habermas, Jürgen, The Structural Transformation of the Public Sphere: An Inquiry into a Category of Bourgeois Society, trad. de T. Burger, Cambridge, MIT Press, 1991.

Hinnebush, Raymond y Anoushiravan Ehteshami (eds.), The Foreign Policies of Middle East States, Londres, Lynne-Rienner, 2002.

Kamrava, Mehran, "Royal Factionalism and Political Liberalization in Qatar”, The Middle East Journal, 63 (2009), pp. 401-420. 
Kamrava, Mehran, "Mediation and Qatari Foreign Policy", The Middle East Journal, 65 (2011), pp. 539-556.

Kamrava, Mehran, Qatar: Small State, Big Politics, Ithaca, Cornell University Press, 2013.

Kawakibi, Salam, "Al-Yazira y la política exterior de Qatar", Awraq. Revista de Análisis y Pensamiento sobre el Mundo Árabe e Islámico Contemporáneo, 2010, núm. 2, pp. 61-78.

Khalaf, Roula y Abigail Fielding-Smith, "How Qatar Seized Control of the Syrian Revolution", The Financial Times, 17 de mayo de 2013, en https:/ / www.ft.com/content/, consultado el 27.VII.2017.

Khalidi, Rashid, "The Origins of Arab Nationalism: Introduction”, en Rashid Khalidi, Lisa Anderson, Muhammad Muslih y Reeva S. Simon (eds.), The Origins of Arab Nationalism, Nueva York, Columbia University Press, 1991.

Korany, Bahgat y Ali E. Hilal Dessouki (eds.), The Foreign Policies of Arab States: The Challenge of Globalization, Cairo, The American University in Cairo Press, 2008.

Lynch, Marc, "Jordan's Identity and Interests", en Shibley Telhami y Michael Barnett (eds.), Identity and Foreign Policy in the Middle East, Ithaca, Cornell University Press, 2002.

Lynch, Marc, Voices of the New Arab Public: Iraq, Al Jazeera, and Middle East Politics Today, Nueva York, Columbia University Press, 2007.

Maguire, Kevin y Andy Lines, "Exclusive: Bush Plot to Bomb His Arab Ally", The Daily Mirror, 22 de noviembre de 2005, en http://www.mirror.co.uk/news/

Miles, Hugh, Al-Jazeera: The Inside Story of the Arab News Channel that is Challenging the West, Nueva York, Grove Press, 2005.

OnLey, James, The Arabian Frontier of the British Raj: Merchants, Rulers, and the British in the Nineteen-Century Gulf, Nueva York, Oxford University Press, 2007.

Patel, David Siddharta, "Repartitioning the Sykes-Picot Middle East? Debunking Three Myths", Middle East Brief, 2016, núm. 103, pp. 1-10.

Peterson, J. E., "Qatar and the World: Branding for a MicroState”, The Middle East Journal, 60 (2006), pp. 732-748. 
PoDen, Elie, The Decline of Arab Unity: The Rise and Fall of the United Arab Republic, Sussex, Academic Press, 1999.

Priego, Alberto, "Las Primaveras Árabes: la influencia de Qatar y sus relaciones con los estados del Golfo", Revista UNISCI, 2015, núm. 39, pp. 233-252.

Provence, Michael, The Great Syrian Revolt and the Rise of Arab Nationalism, Austin, University of Texas Press, 2005.

"Qatari fm Denies Reports on Meshaal Departure from Doha", $\mathrm{Ku}$ wait News Agency, 12 de enero de 2015.

"Qatari Premier Calls for «Clear-Cut» Stand on Israeli «Aggression»”, BBC_Monitoring Middle East, 13 de enero de 2009.

RaBi, Uzi, "Qatar's Relations with Israel: Challenging Arab and Gulf Norms", The Middle East Journal, 63 (2009), pp. 443-459.

SheEHI, Stephen, Foundations of Modern Arab Identity, Gainesville, University Press of Florida, 2004.

Sher wood, Harriet, "Palestine Papers Provoke Anger on Streets of West Bank and Gaza", The Guardian, 25 de enero de 2011, en https:/ / www.theguardian.com/world/2011

SoAge, Ana Belén, "Shaykh Yusuf al-Qaradawi: Portrait of a Leading Islamic Cleric”, Middle East Review of International Affairs, 12 (2008), pp. 51-68.

Solomon, Jay y Nour Malas, "Qatar's Ties to Militants Strain Alliance”, The Wall Street Journal, 23 de febrero 2015, en https:// www.wsj.com/articles

State of Qatar, "Statement before the 60th Session of the General Assembly of the United Nations", Nueva York, 19 de septiembre de 2005.

Telhami, Shibley y Michael Barnett (eds.), Identity and Foreign Policy in the Middle East, Ithaca, Cornell University Press, 2002.

"UAE Summons Qatari Ambassador over Cleric's Comments", Arabian Business. Com, 2 de febrero de 2014.

Ulrichsen, Kristian Coates, Qatar and the Arab Spring, Londres, Hurst \& Co Publishers, 2014.

Ulrichsen, Kristian Coates, The Gulf States in International Political Economy, Nueva York, Palgrave Macmillan, 2016.

WoERTZ, Eckart, "Qatar y el descuido europeo de la región del Golfo Pérsico", Notes Internationals CIDOB, 2012, núm. 46, pp. 1-6. 